\title{
Design and Analysis of a 10× Optical Zoom System for an LWIR Camera
}

\author{
Chang-Min Ok and Sung-Chan Park* \\ Department of Physics, Dankook University, Cheonan 330-714, Korea
}

(Received August 26, 2014 : accepted September 2, 2014)

\begin{abstract}
This paper presents the design and evaluation of the optical zoom system for an LWIR camera. The operating wavelength range of this system is from $7.7 \mu \mathrm{m}$ to $12.8 \mu \mathrm{m}$. Through a paraxial design and optimization process, we have obtained the extended four-group inner-focus zoom system with focal lengths of 10 to $100 \mathrm{~mm}$, which consists of the six lenses including four aspheric surfaces and two diffractive surfaces. The diffractive lenses were used to balance the higher-order aberrations, and its diffraction properties were evaluated by scalar diffraction theory. We have calculated the polychromatic integrated diffraction efficiency and the MTF drop generated by background noise. The f-number of the zoom system is $\mathrm{F} / 1.4$ at all positions. Fields of view are given by $51.28^{\circ} \times 38.46^{\circ}$ at wide field and $5.50^{\circ} \times 4.12^{\circ}$ at narrow field positions. In conclusion, this design procedure results in a $10 \times$ compact zoom lens system useful for an LWIR camera.
\end{abstract}

Keywords : Long wave infrared, Diffractive lens, Zoom lens, Zoom locus

OCIS codes : (220.3620) Lens system design; (220.0220) Optical design and fabrication; (050.1965) Diffractive lenses; (110.3080) Infrared imaging

\section{INTRODUCTION}

A thermal imaging camera has been used to view a target at night for military purposes for the past 40 years. In recent times, civilian demands for the thermal camera have been gradually increasing. Civilian demands are primarily in the security, industry, research and medical fields. The thermal imaging camera measures the radiation of the target, and the measured radiation can be expressed by the distribution of the radiative temperature [1]. It is often necessary for optical systems to have multiple fields of view. A zoom system can have continuous changes of the field of view by moving lens groups along an optical axis.

In this paper, paraxial studies and lens modules are used to obtain the optimum initial design of a $10 \times$ extended four-group inner-focus zoom system for an LWIR camera. It is integrated into uncooled IR detectors with $384 \times 288$ pixels, of which pixel size is $25 \mu \mathrm{m}$. The f-number of the optical zoom system was estimated to be F/1.4 from the Airy disk and MRTD(Minimum Resolvable Temperature Difference) analysis [2-5].

Through an optimization process, we have obtained an optical zoom system satisfying the requirements for a $10 \times$
LWIR camera. In order to balance the wave front aberrations, we located the diffractive surfaces at L3 and L6. Since the diffractive lenses are designed to be very weakly powered and the wavelength to zone period ratio is very small across the entire lens, the scalar predictions of diffraction efficiency are valid to calculate the polychromatic integrated diffraction efficiency. The changes of the MTF(Modulation Transfer Function) due to diffraction efficiency are evaluated from the polychromatic integrated efficiency [6].

\section{OPTICAL DESIGN REQUIREMENTS}

The main requirements of an LWIR zoom system are listed in Table 1. Since the f-number has an effect on Airy disk diameter as well as on MRTD, the optical system with uncooled IR detector should have a low f-number, if possible [7]. Basically spatial resolution of an IR optical system is decided by the specification of IR detector, because target acquisition performance is based on the number of line pairs resolvable across a target critical dimension. Consequently an IR optical system should be designed to have the diffraction limited performance [3].

\footnotetext{
*Corresponding author: scpark@dankook.ac.kr
} 
Design and Analysis of a 10× Optical Zoom System for an LWIR Camera - Chang-Min Ok and Sung-Chan Park 575

TABLE 1. Optical requirements for a LWIR zoom system

\begin{tabular}{c|c}
\hline \hline Parameters & Requirements \\
\hline Spectral range & $7.7 \sim 12.8 \mu \mathrm{m}$ \\
\hline Effective focal length & $10.0 \sim 100.0 \mathrm{~mm}$ \\
\hline F-number & 1.4 at all positions \\
\hline Fields of view at infinity & Wide $: 51.28^{\circ} \times 38.46^{\circ}$ \\
& Narrow $: 5.50^{\circ} \times 4.12^{\circ}$ \\
\hline Instantaneous fields & Wide $: 2.331 \mathrm{mrad}$ \\
of view at infinity & Narrow : $0.250 \mathrm{mrad}$ \\
\hline Pixel format & $384 \times 288$ pixels $(25 \mu \mathrm{m} \mathrm{pitch})$ \\
\hline Detector size & $9.6(\mathrm{H}) \times 7.2(\mathrm{~V}) \mathrm{mm}$ \\
\hline
\end{tabular}

The Airy disk diameter is given by $2.44 \cdot \lambda \cdot F$-number. In diffraction limited system, a pixel's diagonal size of detector should be equal to the Airy disk diameter as follows:

$$
\frac{\lambda F / \#}{d}=\frac{\sqrt{2}}{2.44}=0.58
$$

where $d$ is a pixel width of the detector, $\lambda$ is an operating wavelength, and F/\# is an f-number of the optical system. For a detector with pixel width of $25 \mu \mathrm{m}$, from Eq. (1), the target f-number of the optical zoom system is given by $\mathrm{F} / 1.4$ at central wavelength of $10.2 \mu \mathrm{m}$.

The MRTD is one of the criteria denoting optical system performances, which characterizes the thermal sensitivity at a given spatial frequency [8], whereas the MTF measures the attenuation in modulation depth without regard for thermal property of an object. This MRTD is given by

$$
\operatorname{MRTD}\left(\xi_{t}\right)=A \cdot \frac{(F / \#)}{D^{*} \cdot \operatorname{MTF}\left(\xi_{t}\right)},
$$

where $A$ is a proportional constant depending on the detector, $D^{*}$ is the normalized detectivity of which the average value is known as $1.44 \times 10^{8} \mathrm{cmHz}^{1 / 2} \mathrm{~W}^{-1}$ [9], and $\operatorname{MTF}\left(\xi_{t}\right)$ is the modulation transfer function of the optical system at target frequency of $\xi_{t}$. For a given set of parameters, the MRTD can be calculated from the Eq. (2) [8]. The relationships between the f-number and MRTD are summarized in Table 2.

Since the diffraction MTF depends on the f-number of an optical system, a large f-number results in a large value of MRTD, which reduces the resolution for resolvable temperature difference. Meanwhile, a small f-number gives a good value of MRTD, however, the large aperture system is limited by the cost and the detector. From the exact evaluation for the relationship between f-number and MRTD, optimum f-number of this system is confirmed to be 1.4. The MRTD at F/1.4 is so small that it may give enough resolution.

\section{INITIAL DESIGN FOR AN LWIR ZOOM SYSTEM}

Figure 1 illustrates an extended four-group inner-focus zoom system that has the same total track length at all zoom positions. The zoom system is composed of fixed three groups, the second group for zooming, and the fourth group for focusing.

The powers of the groups are denoted by $k_{1}, k_{2}, k_{3}, k_{4}$,

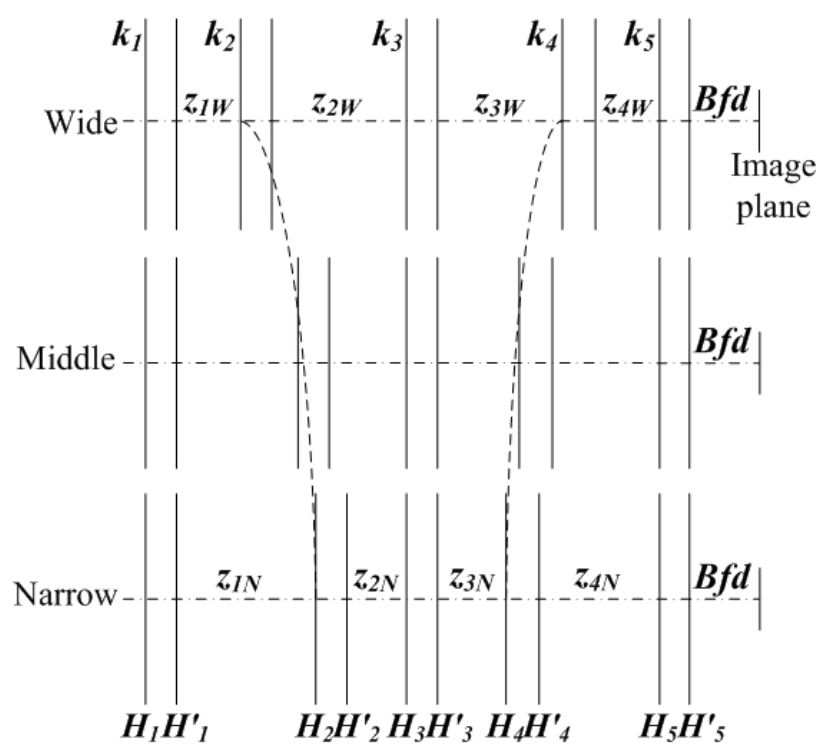

FIG. 1. Layout of an extended four-group inner-focus zoom system.

TABLE 2. F-number versus MRTD

\begin{tabular}{c|c|c|c|c|c}
\hline \hline $\mathrm{F} / \#$ & $\begin{array}{c}\text { Airy disk diameter } \\
{[\mathrm{mm}]}\end{array}$ & $\begin{array}{c}\text { Cut-off frequency } \\
{[\text { cycles } / \mathrm{mm}]}\end{array}$ & $\begin{array}{c}\text { Nyquest frequency } \\
{[\text { cycles/mm] }}\end{array}$ & $\begin{array}{c}\text { Diffraction MTF } \\
{[\text { arb. unit] }}\end{array}$ & $\begin{array}{c}\text { MRTD } \\
\left(\times 10^{-9} \mathrm{~A}\right)\end{array}$ \\
\hline 0.5 & 0.012 & 195 & 20.0 & 0.90 & 3.87 \\
\hline 1.0 & 0.025 & 98 & 20.0 & 0.80 & 8.73 \\
\hline 1.4 & 0.035 & 70 & 20.0 & 0.71 & 13.6 \\
\hline 1.5 & 0.037 & 65 & 20.0 & 0.69 & 15.0 \\
\hline 2.0 & 0.050 & 49 & 20.0 & 0.59 & 23.5 \\
\hline
\end{tabular}


and $k_{5}$, respectively. The distances between the adjacent principal planes are represented by $z_{i W}$ and $z_{i N}(\mathrm{i}=1,2,3$, 4) at wide and narrow field positions, respectively. The first, the third, and the fifth groups are always fixed. While the second group moves to have a longer focal length, the fourth group should move to keep the image position stationary. For the zoom system having zoom ratio of $10 \times$, it is desired that the powers of all groups are positive, except the negative second group for zooming.

We can analytically derive the set of zoom equations for an extended four-group inner-focus zoom system with infinite object by using the Gaussian brackets. The initial design of this zoom system can be formulated as follows [10-12]:

$$
\begin{aligned}
& {\left[k_{1},-z_{1}, k_{2},-z_{2}, k_{3},-z_{3}, k_{4},-z_{4}, k_{5}\right]=K,} \\
& {\left[k_{1},-z_{1}, k_{2},-z_{2}, k_{3},-z_{3}, k_{4},-z_{4}\right]=K b f d,}
\end{aligned}
$$

where $K$ is the optical power at a zoom position. Equations (3) (4) can be rearranged as follows:

$$
\begin{gathered}
k_{1}+k_{2}-z_{1} k_{1} k_{2}+\left(K b f d-K b f d k_{5} z_{4}+K z_{4}+K z_{3}-K b f d k_{5} z_{3}\right) k_{3} \\
+\left(K b f d-K b f d k_{5} z_{4}+K z_{4}\right) k_{4}+\left(-K b f d z_{3}+K b f d k_{5} z_{4} z_{3}-K z_{4} z_{3}\right) k_{3} k_{4}
\end{gathered}
$$

$\left(z_{1}+z_{2}\right) k_{1}+z_{2} k_{2}+\left(-z_{1} z_{2}\right) k_{1} k_{2}+\left(-K b f d z_{3}+K b f d k_{5} z_{4} z_{3}-K z_{4} z_{3}\right) k_{4}$

$$
=1-K b f d+K b f d k_{5} z_{4}-K z_{4}-K z_{3}+K b f d k_{5} z_{3} .
$$

Therefore, Eqs. (5) and (6) can be expressed in a matrix form at wide and narrow field positions as follows:

$$
\left[\begin{array}{llllll}
a_{11} & a_{12} & a_{13} & a_{14} & a_{15} & a_{16} \\
a_{21} & a_{22} & a_{23} & a_{24} & a_{25} & a_{26} \\
a_{31} & a_{32} & a_{33} & a_{34} & a_{35} & a_{36} \\
a_{41} & a_{42} & a_{43} & a_{44} & a_{45} & a_{46}
\end{array}\right]\left[\begin{array}{c}
k_{1} \\
k_{2} \\
k_{1} k_{2} \\
k_{3} \\
k_{4} \\
k_{3} k_{4}
\end{array}\right]=\left[\begin{array}{c}
a_{17} \\
a_{27} \\
a_{37} \\
a_{47}
\end{array}\right]
$$

where $a_{i j}$ is given as a function of $a_{i j}\left(K_{W}, K_{N}, k_{5}, z_{1 W}\right.$, $z_{2 W}, z_{3 W}, z_{4 W}, z_{1 N}, z_{2 N}, z_{3 N}, z_{4 N}, b f d$ ) with $K_{W}$ and $K_{N}$ being the optical powers at wide and narrow field positions, respectively. If the Gauss-Jordan elimination method is applied, Eq. (7) is replaced by

$$
\left[\begin{array}{llllll}
b_{11} & b_{12} & b_{13} & b_{14} & b_{15} & 1 \\
b_{21} & b_{22} & b_{23} & b_{24} & 1 & 0 \\
b_{31} & b_{32} & b_{33} & 1 & 0 & 0 \\
b_{41} & b_{42} & 1 & 0 & 0 & 0
\end{array}\right]\left[\begin{array}{c}
k_{1} \\
k_{2} \\
k_{1} k_{2} \\
k_{3} \\
k_{4} \\
k_{3} k_{4}
\end{array}\right]=\left[\begin{array}{c}
b_{17} \\
b_{27} \\
b_{37} \\
b_{47}
\end{array}\right]
$$

where $b_{i j}$ is given as a function of $a_{i j}$. In order to derive the unknown quantities of $k_{1}, k_{2}, k_{3}$, and $k_{4}$, four equations are obtained from Eq. (8):

$$
\begin{aligned}
& k_{2}=\left(b_{47}-b_{41} k_{1}\right) /\left(b_{42}+k_{1}\right), \\
& k_{3}=\left(A_{1} k_{1}^{2}+A_{2} k_{1}+A_{3}\right) /\left(b_{42}+k_{1}\right), \\
& k_{4}=\left(B_{1} k_{1}^{2}+B_{2} k_{1}+B_{3}\right) /\left(b_{42}+k_{1}\right) .
\end{aligned}
$$

Inserting the conditions of Eqs. (9) (11) into the first row equation of Eq. (8) results in an expression for the unknown parameter $k_{1}$ :

$$
A k_{1}^{4}+B k_{1}^{3}+C k_{1}^{2}+D k_{1}+E=0,
$$

where $A_{i}(i=1,2,3), B_{i}(i=1,2,3), A, B, C, D$ and $E$ are given as a function of $b_{i j}$. Solving Eq. (12) for a given $k_{5}$, we can get $k_{1}$. As a result, $k_{2}, k_{3}$, and $k_{4}$ are determined from Eqs. (9) (11).

For the initial design of a $10 \times$ extended four-group inner-focus system, we input the proper distances between principal points and the targeted total powers at wide and narrow positions. These starting values of $z_{1}, z_{2}, z_{3}, z_{4}$, and $b f d$ are empirically selected to satisfy the basic requirements by using paraxial studies, as shown in Table 3 .

By inputting these starting values of Table 3 into Eqs. (5) and (6), we can obtain the proper values for the power of each group from Eqs. (9) (12). Figures 2 illustrates the four solution sets of Eq. (12) for various $k_{5}$.

In the solutions of case 1 and case 2, the powers of the second group $\left(k_{2}\right)$ are positive. Since the second group is the variator, these positive powers of this group are not valid. In the solutions of case 3 , the powers of the third group are negative, so they are also not valid to an extended four-group inner-focus zoom system. Also, the powers of the second group are so small that the displacement amount of this group is to be larger, not compact in the high zoom ratio system.

Finally, the solutions of case 4 satisfy the requirements for power distribution available to our zoom system having zoom ratio of $10 \times$. Among the solutions of case 4, we took the powers of $k_{1}, k_{2}, k_{3}, k_{4}$, and $k_{5}$, maintaining the most stable zooming locus. Table 4 lists the paraxial design data for the powers obtained from above process.

We have set up a zoom lens system with five thin-lens modules, for which the power of each group and the zoom

TABLE 3. The starting parameters for zoom loci (in $\mathrm{mm}$ )

\begin{tabular}{c|c|c|c|c|c|c}
\hline \hline Position & $z_{1}$ & $z_{2}$ & $z_{3}$ & $z_{4}$ & $b f d$ & $\mathrm{~K}\left[\mathrm{~mm}^{-1}\right]$ \\
\hline Wide & 26.4 & 68.9 & 52.8 & 28.7 & 15.0 & 0.1 \\
\hline Narrow & 91.4 & 3.9 & 28.0 & 53.5 & 15.0 & 0.01 \\
\hline
\end{tabular}




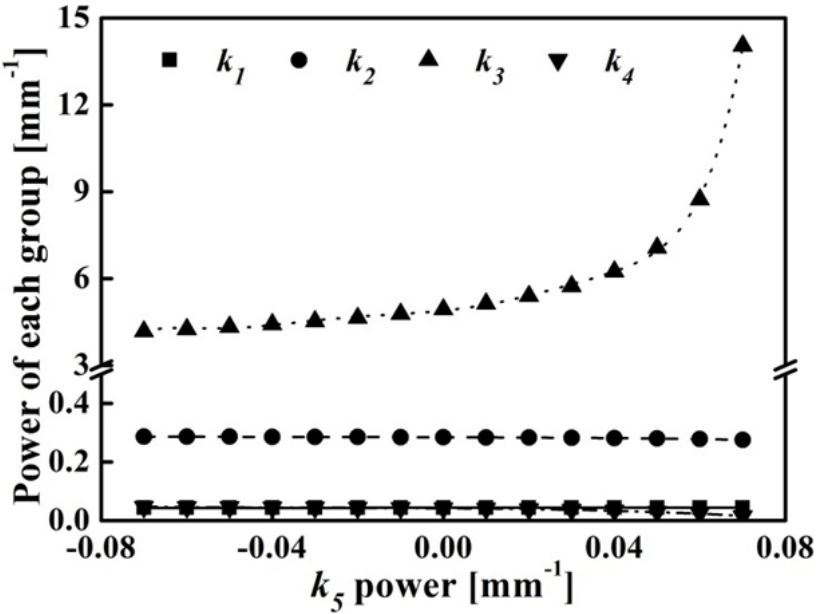

(a) case 1

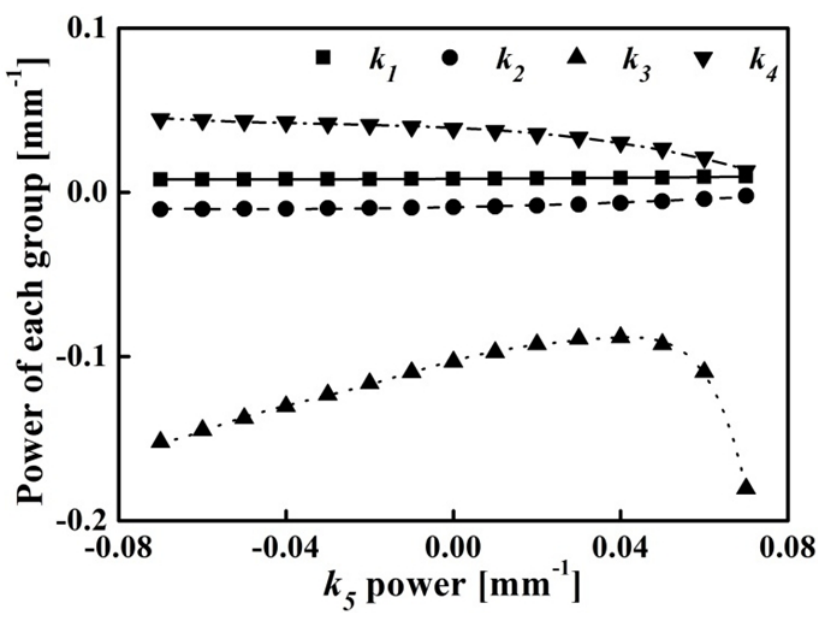

(c) case 3

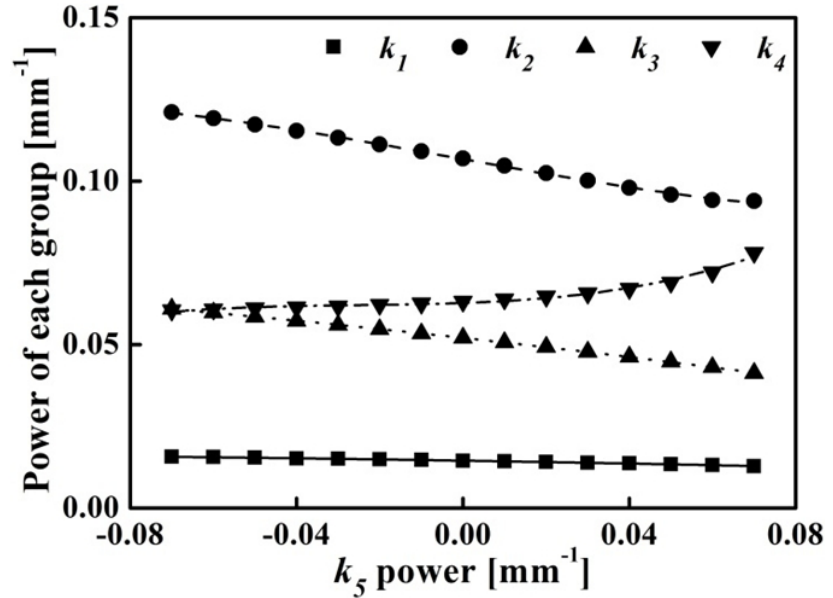

(b) case 2

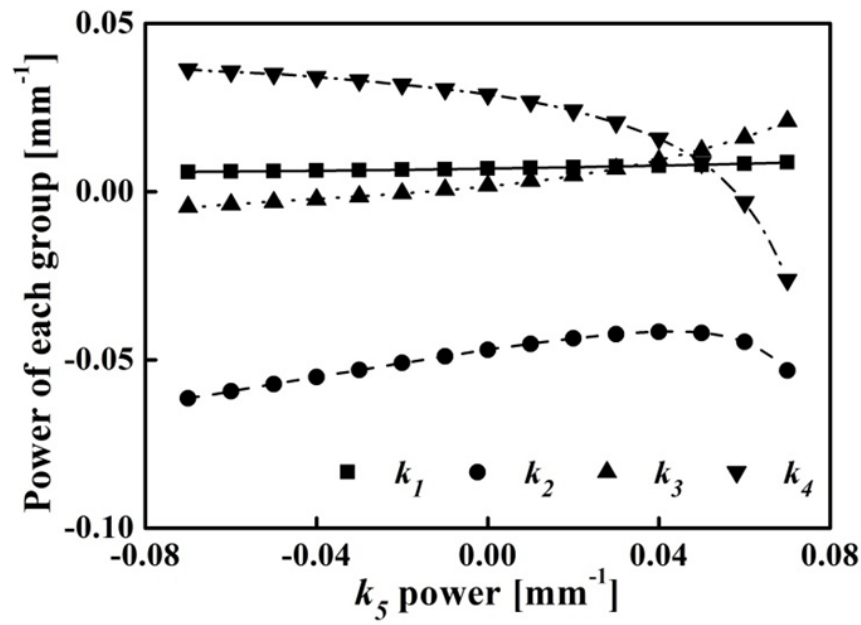

(d) case 4

FIG. 2. Solution sets of the fourth-order equation of Eq. (12) for various $k_{5}$.

TABLE 4. Paraxial design data for the powers (in $\mathrm{mm}^{-1}$ )

\begin{tabular}{c|c|c|c|c|c}
\hline \hline Group & $k_{1}$ & $k_{2}$ & $k_{3}$ & $k_{4}$ & $k_{5}$ \\
\hline Powers & 0.0071 & -0.0447 & 0.0038 & 0.0261 & 0.0131 \\
\hline
\end{tabular}

locus inputs are taken from Table 4 and Table 3. The lens modules do not reflect higher-order aberrations, so reducing the aperture and the field size of the system is desirable $[13,14]$. Thus, we took a zoom system with a half image size of $3 \mathrm{~mm}$ and f-number of $F / 3$ at all positions. The air distances between modules were constrained to be longer than $3.0 \mathrm{~mm}$ for the mounting space.

In Table 3 and Table 4 of the previous paraxial study, the thickness of each group and the air spaces are not presented. They can be derived by specifying the design variables of the lens modules, such as the effective focal length $\left(F L_{M}\right)$, the front $\left(F F_{M}\right)$ and the back focal lengths $\left(B F_{M}\right)$, and the air spaces between the modules. Figure 3 shows the initial zoom design composed of five thick lens modules obtained from this process. The focal lengths range from 10.0 to $100.0 \mathrm{~mm}$. Table 5 shows the first-order properties and the zoom loci at each position, and Table 6 lists the design data for each module. In Table $5, d_{i j}$ $(j=1,2,3,4)$ are the air spaces between the modules at the $i$-th zoom position.

A real lens group is generally composed of several lens elements, which should be equivalent to the lens module given in Table 6. In this paper, an optimization design method is used to design a real lens group equivalent to the module of each group. We choose an appropriate structure for each real lens group and scale it up or down so that the focal length of each real lens group is the same as that of the lens module. By using the optimization method of Code- $\mathrm{V}$, we matched the first-order quantities of the lens module to those of a real lens. For the conversion process, the design variables of the real lens are the radius of each surface, the thickness, and the refractive index. Therefore, the three constraints given in Table 6 can be 
satisfied by specifying the lens design variables. After a few iterations, the real lenses of the groups are obtained.

If a zoom system equivalent to the lens module zoom system is to be achieved, the air spaces $\left(d_{j i}\right)$ between groups should be set according to the zoom loci of Table 5 at each position. This procedure results in a zoom system

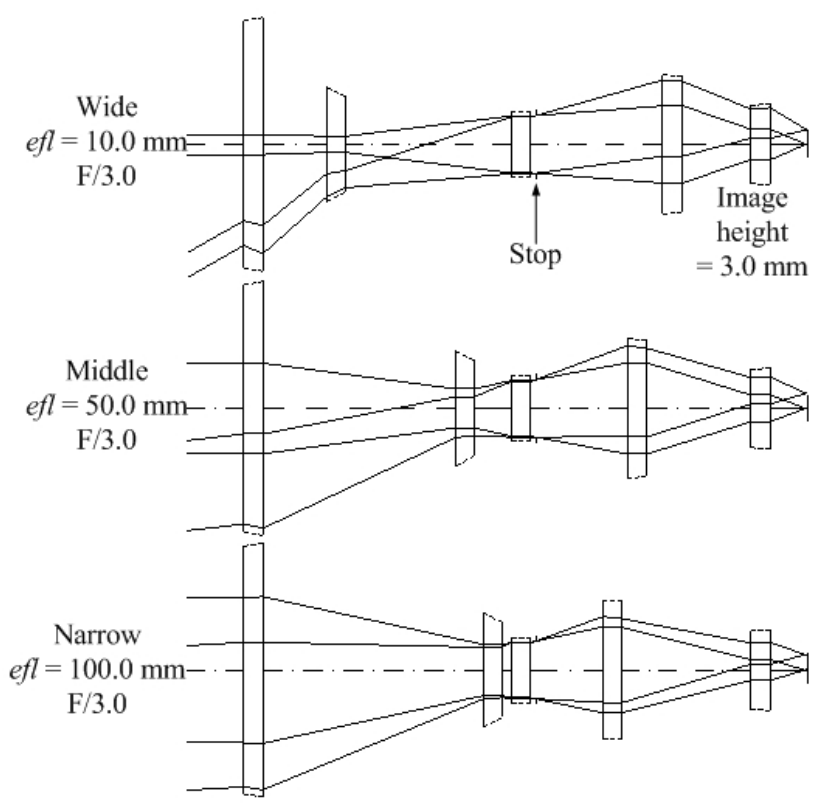

FIG 3. Optimized zoom system consisting of five thick lens modules.

TABLE 5. First-order properties and zooming loci of the zoom system consisting of five thick lens modules (in mm)

\begin{tabular}{c|c|c|c}
\hline \hline Parameters & Wide & Middle & Narrow \\
\hline $\begin{array}{c}\text { Effective focal } \\
\text { length }\end{array}$ & 10.0 & 50.0 & 100.0 \\
\hline $\begin{array}{c}\text { Back focal } \\
\text { length }\end{array}$ & 15.0 & 14.7 & 14.3 \\
\hline $\begin{array}{c}\text { Front focal } \\
\text { length }\end{array}$ & 66.7 & 291.7 & 381.4 \\
\hline$d_{1 i}$ & 26.7 & 81.4 & 93.2 \\
\hline$d_{2 i}$ & 69.8 & 15.1 & 3.3 \\
\hline$d_{3 i}$ & 53.5 & 38.7 & 28.4 \\
\hline$d_{4 i}$ & 29.1 & 43.9 & 54.2 \\
\hline
\end{tabular}

TABLE 6. Design data for the lens modules in the optimized lens module zoom system (in mm)

\begin{tabular}{c|r|r|r|r|r}
\hline \hline Parameter & Group 1 & Group 2 & Group 3 & Group 4 & Group 5 \\
\hline$F L_{M}$ & 142.76 & -22.67 & 226.73 & 38.83 & 77.38 \\
\hline$B F_{M}$ & 133.58 & -23.00 & 226.32 & 36.92 & 73.58 \\
\hline$F F_{M}$ & -150.03 & 23.46 & -217.60 & -38.48 & -78.32 \\
\hline
\end{tabular}

equivalent to the lens module zoom system within paraxial optics. From the evaluation of this zoom system, the agreement of the first-order quantities between the two systems is good.

\section{OPTIMIZED DESIGN FOR A ZOOM SYSTEM USING DIFFRACTIVE LENSES}

In the initial design of the zoom system, we reduced the aperture and the field size so that they were too small. If current specifications for an LWIR zoom camera are to be met, the aperture and the field size should be increased. The f-numbers are extended to $F / 1.4$ at all positions. The half image size should be $6.0 \mathrm{~mm}$ for an uncooled IR detector. In an extended aperture and field system, however, the aberrations that were not corrected in the previous design become significant. In order to improve the overall performance of the zoom system, we balance the aberrations of the starting data. In this process, the first-order layouts are fixed, and the residual aberrations are corrected using aspheric surfaces and diffractive lenses. Finally, a zoom system having good performance was obtained, and its layout is shown in Fig. 4. Table 7 lists the design data.

This system consists of the six lenses including two diffractive surfaces. The diffractive surfaces are used to balance the wave-front aberrations. They are located in the front surface of L3 and the back surface of L6. The hybrid lenses such as L3 and L6 have refractive and diffractive properties at the same time. Therefore the hybrid lens differs from a conventional refractive lens in that it can produce many images due to diffraction orders. These

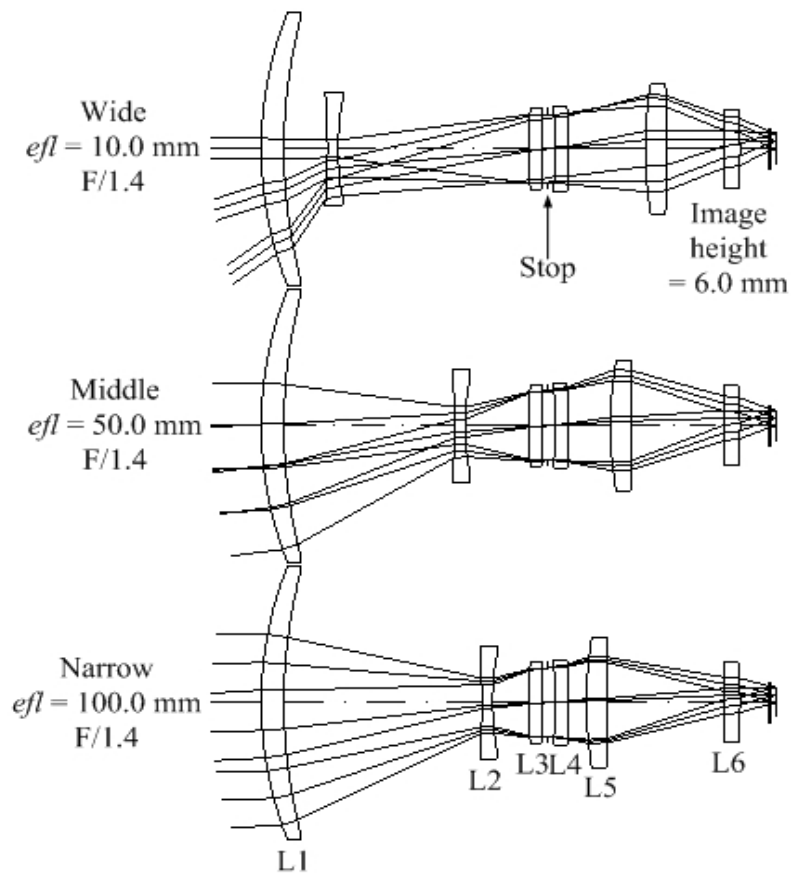

FIG. 4. Layout of an optimized zoom system. 
Design and Analysis of a 10× Optical Zoom System for an LWIR Camera - Chang-Min Ok and Sung-Chan Park 579

TABLE 7. Design data of an optimized zoom system (in mm)

\begin{tabular}{c|c|c|c}
\hline \hline Surfaces & Radius & Thickness & Materials \\
\hline 1 & 136.73 & 9.5 & $\mathrm{Ge}$ \\
\hline 2 & 191.8 & $18.28 \sim 83.28$ & \\
\hline 3 & A -207.92 & 4.5 & $\mathrm{Ge}$ \\
\hline 4 & 97.2 & $80 \sim 15$ & \\
\hline 5 & A, (D) 138.75 & 6 & $\mathrm{ZnSe}$ \\
\hline 6 & -560.69 & 2 & \\
\hline $7($ stop) & Infinity & 3.5 & \\
\hline 8 & -122.85 & 5.5 & $\mathrm{Ge}$ \\
\hline 9 & -202.86 & $32.24 \sim 7.45$ & \\
\hline 10 & A 137.19 & 9 & $\mathrm{Ge}$ \\
\hline 11 & -657.17 & $23.86 \sim 48.65$ & \\
\hline 12 & Infinity & 7 & $\mathrm{ZnSe}$ \\
\hline 13 & A, (D) -119.16 & 12 & \\
\hline 14 & Infinity & 1 & Ge \\
\hline 15 & Infinity & 1.9 & \\
\hline 16 & Infinity & - & \\
\hline
\end{tabular}

(A): aspheric surface, (D): diffractive surface)

diffraction images serve to lower the contrast of the desired image.

The rotationally symmetric diffractive lens has the surfacerelief of ring type. The number of rings $(N)$ on the diffractive surface is given by Eq. (13):

$$
N=\frac{D^{2}}{8 \lambda_{0} f_{0}}
$$

where $D$ is the diameter of diffractive surface and $f_{0}$ is the focal length at design wavelength of $\lambda_{0}$. For a diffractive lens having short focal length, as shown in Eq. (13), it can be seen that $N$ is to be large. Therefore, we need to budget the small power on the diffractive lens. Table 8 gives the specification of the designed hybrid lens in the zoom system. Table 9 lists the radius of each zone, given by $r_{n}=\sqrt{2 n \lambda_{0} f_{0}}$.

Figure 5 compares two point spread functions by L3, Fig. 5(a) is obtained from refracted image only and Fig. 5(b) from refracted plus diffracted images. Other diffraction orders, not design order, generate the background noises such as flares. They result in degrading the contrast at around zero frequency. However, the width of the central peak is much more narrow than that of the refracted image, which eventually improves the image quality at high frequency.

For a diffractive optical system that utilizes spectrally broadband illumination, the wavelength dependence of diffraction efficiency needs to be considered when evaluating imaging performance. To determine the polychromatic
TABLE 8. Specifications of the hybrid lenses (in mm)

\begin{tabular}{c|c|c|c|c}
\hline \hline Lens & $N[\mathrm{EA}]$ & $\begin{array}{c}\text { EFL } \\
\text { (Refractive }+ \\
\text { Diffractive) }\end{array}$ & $\begin{array}{c}\text { EFL } \\
\text { (Diffractive } \\
\text { only) }\end{array}$ & Aperture \\
\hline L3 & 6.459 & 76.115 & 1747.007 & 30.4 \\
\hline L6 & 4.562 & 81.373 & 2009.356 & 27.4 \\
\hline
\end{tabular}

TABLE 9. The radius of each zone $r_{n}$ (in $\mathrm{mm}$ )

\begin{tabular}{c|c|c}
\hline \hline$r_{n}$ & L3 & L6 \\
\hline$r_{1}$ & 5.979 & 6.412 \\
\hline$r_{2}$ & 8.458 & 9.069 \\
\hline$r_{3}$ & 10.358 & 11.110 \\
\hline$r_{4}$ & 11.962 & 12.827 \\
\hline$r_{5}$ & 13.373 & - \\
\hline$r_{6}$ & 14.650 & - \\
\hline
\end{tabular}

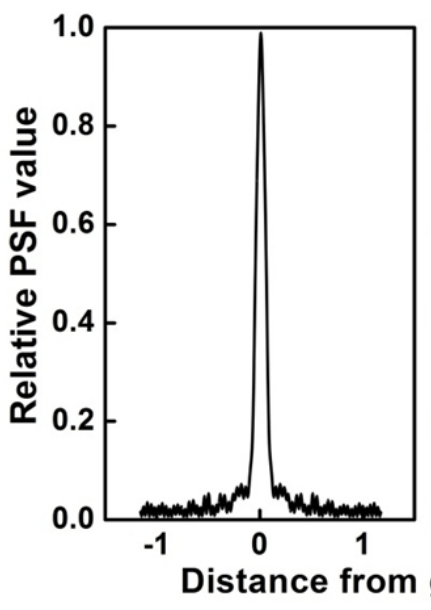

(a)

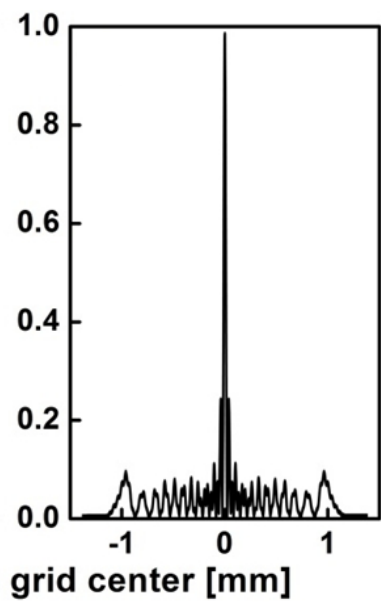

(b)
FIG. 5. Point spread functions by L3 on image plane. (a) refracted image only and (b) refracted plus diffracted image.

transfer function for a diffractive lens, it is first to define a polychromatic integrated efficiency for the wavelength band ranging from $\lambda_{\min }$ to $\lambda_{\max }$. Denoting the integrated efficiency at wavelength $\lambda$ by $\eta_{\text {int }}(\lambda)$, the polychromatic integrated efficiency is given by [6]

$$
\eta_{\text {int.poly }}=\frac{\int_{\lambda_{\min }}^{\lambda_{\max }} \eta_{\text {int }}(\lambda) d \lambda}{\lambda_{\max }-\lambda_{\min }} .
$$

In Table 8 , the diffractive components are very weakly powered, to the $4 \%$ of the hybrid component's powers. In addition to that, the wavelength to zone period ratio is very small across the entire lens, as shown in Table 9. In 
these situations, it is known that scalar predictions of diffraction efficiency are valid to replace the $\eta_{\text {int }}(\lambda)$ of Eq. (14) $[6,15]$. The scalar diffraction theory value for the diffraction efficiency in order $m$ for wavelength $\lambda$ is given by

$$
\eta_{\text {scalar }}(\lambda)=\frac{\sin ^{2}\left[\pi\left(\frac{\lambda_{0}}{\lambda}-m\right)\right]}{\left[\pi\left(\frac{\lambda_{0}}{\lambda}-m\right)\right]^{2}}
$$

where $\lambda_{0}$ is the design wavelength for the diffractive lens. The expression for $\eta_{\text {scalr }}(\lambda)$ given by Eq. (15) cannot be explicitly integrated, but an approximate expression for $\eta_{\text {int }}$.poly can be found by expanding $\eta_{\text {scal }}(\lambda)$ in a power series and integrating term by term. The resulting approximate expression for the first order diffraction of $m$ $=1$ is given by

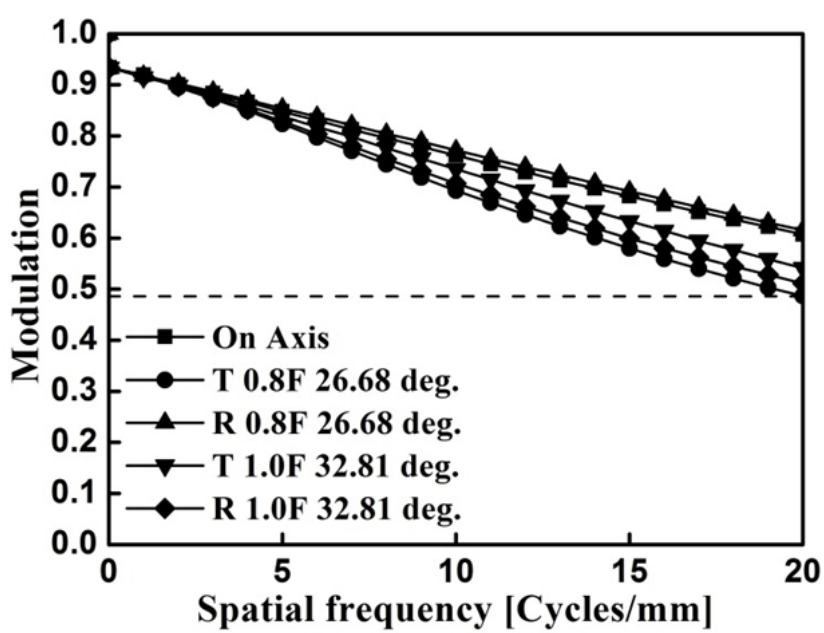

(a)

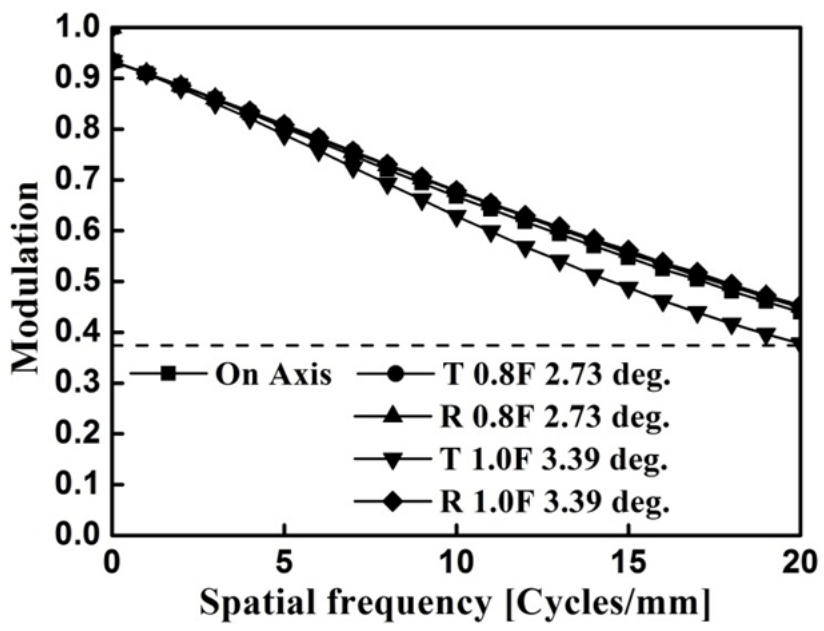

(b)

FIG. 6. Polychromatic MTF calculated by the polychromatic integrated diffraction efficiency. (a) Wide field position, (b) Narrow field position.

$$
\begin{aligned}
\eta_{\text {int.poly }} \cong 1+ & \frac{\pi^{2}}{3 \lambda_{0}}\left(\lambda_{\min }+\lambda_{\max }-\lambda_{0}\right) \\
& -\frac{\pi^{2}}{9 \lambda_{0}^{2}}\left(\lambda_{\min }^{2}+\lambda_{\min } \lambda_{\max }+\lambda_{\max }^{2}\right) .
\end{aligned}
$$

As a result, the polychromatic integrated efficiency of an LWIR zoom system is $93.39 \%$ and background noise reduces the diffraction efficiency by $6.61 \%$ for $\lambda_{\min }=7.7 \mu \mathrm{m}$, $\lambda_{\max }=12.8 \mu \mathrm{m}$, and $\lambda_{0}=10.2 \mu \mathrm{m}$. These losses in diffraction efficiency may reduce the contrast of image. Figure 6 shows the polychromatic modulation transfer functions calculated by using the Eq. (16). The polychromatic MTF is scaled by $\eta_{\text {int }}$-poiy and drops down at zero-frequency. The resulting curves show the behavior predicted by point spread functions of Fig. 5(b).

\section{CONCLUSION}

We have designed and evaluated the optical zoom system for an LWIR camera with an uncooled IR detector. The fields of view are $51.28^{\circ} \times 38.46^{\circ}$ at wide and $5.50^{\circ}$ $\times 4.12^{\circ}$ at narrow field positions. The optimum f-number of the zoom system was determined to be F/1.4 from Airy disk and MRTD analysis.

Through paraxial design and optimization method, we have obtained an extended four-group inner-focus zoom system having a focal length of 10 to $100 \mathrm{~mm}$. Balancing the wave-front aberrations by use of diffractive surfaces, we improved the performance of the zoom system further. To evaluate the polychromatic transfer function for a weakly powered diffractive lens, the scalar diffraction theory was effectively used.

As a result, the polychromatic integrated efficiency is 93.39\% and background noise reduces the diffraction efficiency by $6.61 \%$. This loss in diffraction efficiency slightly reduced the contrast of image at zero-frequency, and scaled down the polychromatic MTF by $\eta_{\text {int }}$.poly. However, the designed optical zoom system has enough image qualities for an LWIR camera, as shown in Fig. 6. Consequently, the zoom system developed in this work performs reasonably as an LWIR zoom camera.

\section{REFERENCES}

1. J. M. Loyd, Thermal Imaging System (Plenum Press, New York, USA, 1975).

2. G. C. Holst, "Imaging system performance based upon F $\lambda / d$," Opt. Eng. 46, 103204 (2007).

3. J. A. Ratches, R. H. Vollmerhausen, and R. G. Driggers. "Target acquisition performance modeling of infrared imaging systems: Past, present, and future," IEEE Sensors J. 1, 31-40 (2001).

4. G. C. Holst, Testing and Evaluation of Infrared Imaging 
Design and Analysis of a 10× Optical Zoom System for an LWIR Camera - Chang-Min Ok and Sung-Chan Park 581

Systems (JCD Publishing, Winter Park, USA, 2008).

5. NVESD Manual and Reference Guide, Rev 7.0 (U.S. Army Night Vision and Electronic Sensors Directorate, USA, 2002).

6. D. A. Buralli and G. M. Morris, "Effects of diffraction efficiency on the modulation transfer function of diffractive lenses," Appl. Opt. 31, 4389-4396 (1992).

7. C. M. Ok, H. J. Lee, and H. K. Kim, "Optimization of a middle infrared optical system with double magnification," in Proc. OSK Summer Meeting (Phoenix Park, Korea, Jul. 2008), pp. 397-398.

8. A. Daniels, Field Guide to Infrared Systems, Detectors, and FPAs (SPIE Press, Bellingham, Washington, USA, 2010).

9. R. K. Bhan, R. S. Saxena, C. R. Jalwania, and S. K. Lomash, "Uncooled infrared microbolometer arrays and their characterisation techniques," Defense Science J. 59, 580-589 (2009).
10. S. C. Park and J. U. Lee, "Rapid optical zoom system design using optimized lens module," J. Korean Phys. Soc. 32, 815-822 (1998).

11. K. Tanaka, "Paraxial theory in optical design in terms of Gaussian brackets," in Process in Optics XXIII, E. Wolf ed. (North-Holland, Amsterdam, The Netherlands, 1986), pp. 63-111.

12. K. Tanaka, "Paraxial analysis of mechanically compensated zoom lenses. 1: Four-component types," Appl. Opt. 21, 2174-2183 (1982).

13. S. C. Park and R. R. Shannon, "Zoom lens design using lens module," Opt. Eng. 35, 1668-1676 (1996).

14. S. C. Park and S. H. Lee, "Zoom lens design for a $10 \mathrm{x}$ slim camera using successive procedures," J. Opt. Soc. Korea 17, 518-524 (2013).

15. D. A. Buralli, G. M. Morris, and J. R. Rogers, "Optical performance of holographic kinoforms," Appl. Opt. 28, 976-983 (1989). 